\title{
Criterion for the Stability of Numerical Integration Methods for the Solution of Systems of Differential Equations*
}

\author{
Abbas I. Abdel Karim ** \\ Senior Lecturer in Mathematics, Faculty of Science, University of Khartoum, Sudan
}

(April 20, 1967)

\begin{abstract}
The problem of studying the growth of the error is most important for the numerical solution of differential equations. In this paper the Wilf's criterion is generalized to be applied for systems of differential equations. A general theorem is investigated and regions of stability have to be determined. The use of an electronic computer is more essential for such regions to be characterized. These regions of stability have the property that, the error introduced at any stage tends to decay. The regions of stability for particular numerical methods are explicitly determined.
\end{abstract}

Key Words: Numerical integration, propagation of error, stability, systems of differential equations.

\section{Introduction}

The system of $m$ ordinary differential equations can be written in the vector form as follows:

$$
Y^{\prime} \equiv \frac{d Y}{d x}=F(x ; Y)
$$

with the initial value $Y\left(x_{0}\right)=Y_{0}$,

where

$$
Y=\left(\begin{array}{c}
y_{1} \\
y_{2} \\
\vdots \\
y_{m}
\end{array}\right),
$$

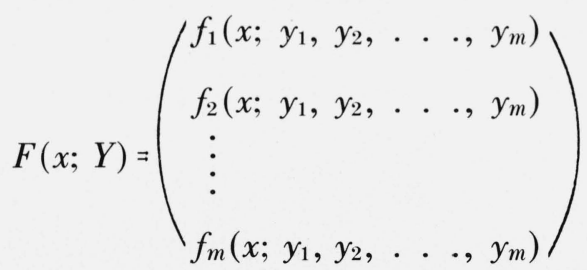

Let the vectors:

$Y(x)$ be the exact solution of the system (1),

$Y_{n}$ be the theoretical (approximate) solution at $x=x_{n}$,

$Y_{n}$ be the practical (computed) solution at $x=x_{n}$.

We define the error $\epsilon_{n}$ of the solution at the point $x_{n}$ by:

$$
\epsilon_{n}=\stackrel{*}{Y}_{n}-Y\left(x_{n}\right) \text {. }
$$

*An invited paper.

**Present address: Faculty of Science, University of Khartoum, Sudan, Khartoum, P.O. Box 321. 
Hence we get,

$$
\begin{aligned}
\epsilon_{n}^{\prime} & =\stackrel{*}{Y_{n}^{\prime}}-Y^{\prime}\left(x_{n}\right) \\
& =F\left(x_{n} ; \stackrel{*}{Y}_{n}\right)-F\left(x_{n} ; \stackrel{*}{Y}_{n}-\epsilon_{n}\right) .
\end{aligned}
$$

Assume that:

(a) $F\left(x_{n} ; Y\right)$ is a continuous function of $Y$ for $Y$ contained in the closed intervals whose endpoints are $Y\left(x_{n}\right)$ and $Y_{n}$,

(b) the matrix $\left(\frac{\partial f_{i}\left(x_{n} ; Y\right)}{\partial y_{j}}\right) ;(i, j=1,2, \ldots, m)$ exists for $Y$ in the open interval whose endpoints are $Y\left(x_{n}\right)$ and $Y_{n}$.

Therefore, by the mean value theorem we get:

$$
\epsilon_{n}^{\prime}=\left(\frac{\partial f_{i}}{\partial y_{j}}\right) \epsilon_{n}=J_{n} \epsilon_{n},(i, j=1,2, \ldots ., m),
$$

where the elements of the Jacobian matrix $J_{n}=\left(\frac{\partial f_{i}}{\partial y_{j}}\right)_{n}$ are taken at suitable places

$$
\left(x_{n}, \stackrel{*}{Y}_{n}-\operatorname{diag}\left(\theta_{i}\right) \cdot \epsilon_{n}\right), 0<\theta_{i}<1, i=1,2, \ldots, m .
$$

TheOREM: Stability criterion for numerical integration methods for the solution of systems of differential equations.

The integration formula

$$
\mathrm{Y}_{\mathrm{n}+1}=\sum_{\nu=-\mathrm{q}}^{0} \mathrm{a}_{\nu} \mathrm{Y}_{\mathrm{n}+\nu}+\mathrm{h} \sum_{\nu=-\mathrm{q}}^{1} \mathrm{~b}_{\nu} \mathrm{Y}_{\mathrm{n}+\nu}^{\prime}
$$

for the solution of $\mathrm{m}$ ordinary differential equations $\mathrm{Y}^{\prime}=\mathrm{F}(\mathrm{x} ; \mathrm{Y})$ is stable if and only if, the Hermitian forms with the coefficients

$$
\mathrm{A}\left(\mathrm{rrs}_{\mathrm{r})}^{(i)}=\sum_{\mathrm{l}=0}^{\min (\mathrm{r}, \mathrm{s})}\left\{\overline{\mathrm{C}}_{\mathbf{q}+\mathrm{l}+1-\mathrm{r}, \mathrm{i}} \mathrm{C}_{\mathbf{q}+1+1-\mathrm{s}, \mathrm{i}}-\mathrm{C}_{\mathbf{r}-1, \mathrm{i}} \overline{\mathrm{C}}_{\mathrm{s}-1, \mathrm{i}}\right\}\right.
$$

for all $\mathrm{i}=1,2, \ldots, \mathrm{m}$ are positive definite

where

$$
\mathrm{C}_{\delta, \mathrm{i}}=\kappa_{\mathrm{i}} \mathrm{b}_{\delta-\mathrm{q}}-\mathrm{a}_{\delta-\mathrm{q}}, \delta=0,1, \ldots, \mathrm{q}+\mathrm{l}
$$

with

$$
\begin{aligned}
& \kappa_{\mathrm{i}}=-\mathrm{h} \rho_{\mathrm{i}}, \mathrm{h} \text { the step of integration, } \mathrm{a}_{1}=-1, \\
& \rho_{\mathrm{i}}=\text { the eigenvalues of the Jacobian matrix }\left(\frac{\partial \mathrm{f}_{\mathrm{i}}}{\partial \mathrm{y}_{\mathrm{j}}}\right) \\
& \mathrm{b}_{1}\left\{\begin{array}{l}
=0 \quad \text { an extrapolation formula } \\
\neq 0 \quad \text { if }(4) \quad \text { an interpolation formula. }
\end{array}\right.
\end{aligned}
$$

Proof: The formula (4) gives the theoretical approximating vector $Y_{n+1}$ for the exact value $Y\left(x_{n}+h\right)$. The computed (practical) solution at $x=x_{n+1}$ is determined as follows: 


$$
\stackrel{*}{Y}_{n+1}=\sum_{\nu=-q}^{0} a_{\nu} \stackrel{*}{Y}_{n+\nu}+h \sum_{\nu=-q}^{1} b_{\nu} \stackrel{*}{Y}_{n+\nu}^{\prime}+R_{n+1}
$$

where $R_{n+1}$ the round off error.

The corresponding exact solution is

$$
Y\left(x_{n}+h\right)=\sum_{\nu=-q}^{0} a_{\nu} Y\left(x_{n}+\nu h\right)+h \sum_{\nu=-q}^{1} b_{\nu} Y^{\prime}\left(x_{n}+\nu h\right)+T_{k+1},
$$

where the truncation error $T_{k+1}=\mu_{k+1} h^{k+1} Y_{\left(x_{n}\right)}^{(k+1)}+0\left(h^{k+2}\right), k \geqslant 1, \mu_{k+1} \neq 0$. Subtracting (8) from (7) we have

$$
\stackrel{*}{Y}_{n+1}-Y\left(x_{n}+h\right)=\sum_{\nu=-q}^{0} a_{\nu}\left(\stackrel{*}{Y}_{n+\nu}-Y\left(x_{n}+\nu h\right)\right)+h \sum_{\nu=-q}^{1} b_{\nu}\left(\stackrel{*}{Y}_{n+\nu}^{\prime}-Y^{\prime}\left(x_{n}+\nu h\right)\right)+R
$$

where $R_{n+1}-T_{k+1}=R$ is generally a nonzero vector.

Substituting (2) and (3) in (9) we get the difference equation for the error

$$
\epsilon_{n+1}=\sum_{\nu=-q}^{0} a_{\nu} \epsilon_{n+\nu}+h \sum_{\nu=-q}^{1} b_{\nu} J_{n+\nu} \epsilon_{n+\nu}+R
$$

where

$$
J_{n+\nu}=\left(\frac{\partial f_{i}}{\partial y_{j}}\right)_{n+\nu}
$$

In studying the local growth of the error $\epsilon_{n}$ it is reasonable to assume that $R$ and the matrices $\left(\frac{\partial f_{i}}{\partial y_{j}}\right)$ in a small interval $\left(x_{-q}, x_{1}\right)$ are both constants and they may be written in the form $\tilde{R}$ and $\tilde{J}$ respectively. In practice they vary slowly from step to step, otherwise the step size of integration is too large, Hamming [2]. ${ }^{1}$ Under these assumptions we get from (10) the nonhomogenous system of linear difference equations with constant real coefficients in the form:

$$
\epsilon_{n+1}=\sum_{\nu=-q}^{0} a_{\nu} \epsilon_{n+\nu}+h \tilde{J} \sum_{\nu=-q}^{1} b_{\nu} \epsilon_{n+\nu}+\tilde{R}
$$

To solve this system, we introduce a new vector variable $\hat{\epsilon}$ in place of the variable $\epsilon$ by means of a nonsingular linear transformation:

$$
\hat{\epsilon}=T^{-1} \epsilon \text {, where } \operatorname{det} T \neq 0 \text {. }
$$

Therefore the system (11) is transformed into:

$$
\hat{\epsilon}_{n+1}=\sum_{\nu=-q}^{0} a_{\nu} \hat{\epsilon}_{n+\nu}+h K \sum_{\nu=-q}^{1} b_{\nu} \hat{\epsilon}_{n+\nu}+\hat{R}
$$

where

$$
T^{-1} \tilde{R}=\hat{R}, \quad \mathrm{~K}=T^{-1} \tilde{J} T .
$$

In particular the transformation $T$ may be chosen so as to put the matrix $T^{-1} \tilde{J} T$ into the classical canonical form, Zurmühl [3].

' Figures in brackets indicate the literature references at the end of this paper. 
The corresponding homogeneous system of (13) has the form

$$
\hat{\epsilon}_{n+1}=\sum_{\nu=-q}^{0} a_{\nu} \hat{\epsilon}_{n+\nu}+h K \sum_{\nu=-q}^{1} b_{\nu} \hat{\epsilon}_{n+\nu}
$$

CASE I: If the eigenvalues $\rho_{i}$ of the matrix $\tilde{J}$ are all distinct then the matrix $K$ has these eigenvalues in the principal diagonal and zeros elsewhere. In this case the system (14) has the form:

$$
\begin{gathered}
\left(\kappa_{i} b_{1}+1\right) \hat{\epsilon}_{i, n+1}=\sum_{\nu=-q}^{0}\left(a_{\nu}-\kappa_{i} b_{\nu}\right) \hat{\epsilon}_{i, n+\nu}, \\
i=1,2, \ldots, m
\end{gathered}
$$

where

$$
\kappa_{i}=-h \rho_{i}
$$

Put $\hat{\epsilon}_{i, \nu}=\lambda_{i}^{\nu}$, Nörlund [4], we get from (15) the characteristic equations:

$$
\begin{gathered}
\left(\kappa_{i} b_{1}+1\right) \lambda_{l}^{q+1}+\sum_{\nu=0}^{q}\left(\kappa_{i} b_{-\nu}-a_{-\nu}\right) \lambda_{l}^{q-\nu}=0, \\
i=1,2, \ldots, m
\end{gathered}
$$

CASE II: If the eigenvalues $\rho_{i}$ of the matrix $\tilde{J}$ are not all distinct, then the matrix $\tilde{J}$ may be transformed to:

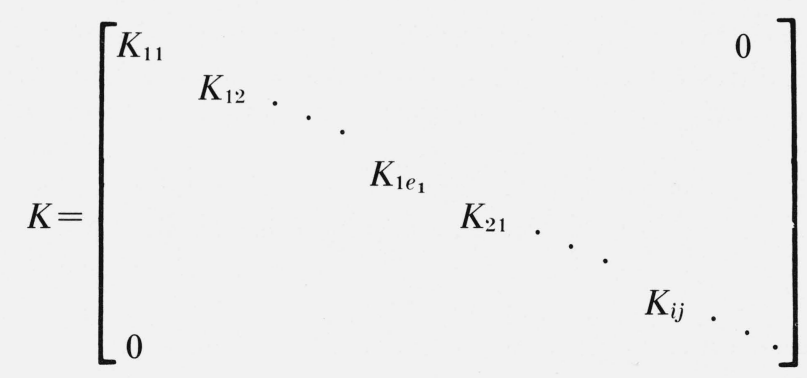

where each submatrix $K_{i j}$ is of the form:

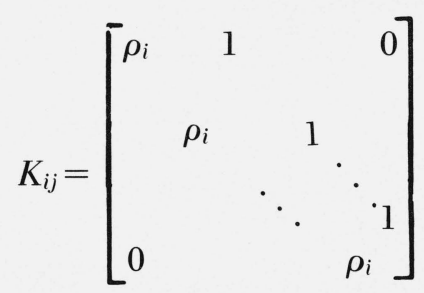

with $\rho_{i}$ in each position in the leading diagonal, unity in each position in the diagonal immediately above it, and with zeros elsewhere. In this case of nondistinct eigenvalues, we have for every submatrix of order $\gamma$ a system of equations in the form: 


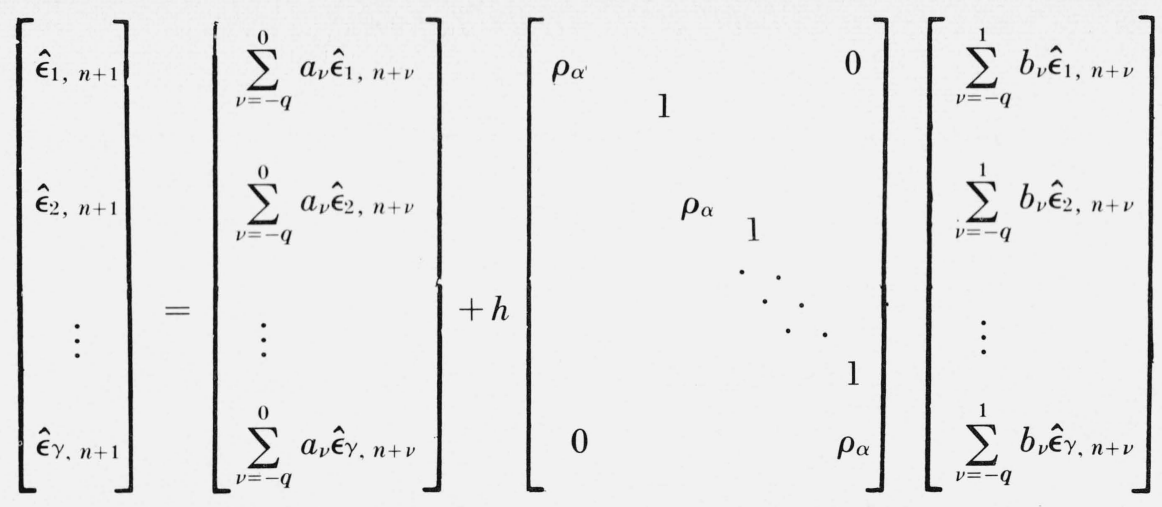

That means

$$
\hat{\boldsymbol{\epsilon}}_{i, n+1}=\sum_{\nu=-q}^{0} a_{\nu} \hat{\boldsymbol{\epsilon}}_{i, n+\nu}+h \sum_{\nu=-q}^{1} b_{\nu}\left\{\boldsymbol{\rho}_{\alpha} \hat{\boldsymbol{\epsilon}}_{i, n+\nu}+\underset{*}{*} \boldsymbol{\epsilon}_{i+1, n+\nu}\right\},
$$

where

$$
i=1,2, \ldots, \gamma, \quad \stackrel{*}{\gamma}=\left\{\begin{array}{lll}
1 & & i<\gamma \\
0 & \text { for } & i=\gamma .
\end{array}\right.
$$

The characteristic equations system can be got as follows:

$$
\begin{gathered}
\left(\kappa_{\alpha} b_{1}+1\right) \lambda_{i}^{q+1}+\sum_{\nu=0}^{q}\left(\kappa_{\alpha} b_{-\nu}-a_{-\nu}\right) \lambda_{i}^{q-\nu}=0, \\
i=1,2, \ldots, \gamma
\end{gathered}
$$

According to (16) and (18) the characteristic equations system has the form:

$$
\begin{gathered}
\left(\kappa_{i} b_{1}+1\right) \lambda_{i}^{q+1}+\sum_{\nu=0}^{q}\left(\kappa_{i} b_{-\nu}-a_{-\nu}\right) \lambda_{l}^{q-\nu}=0 \\
i=1,2, \ldots, m,
\end{gathered}
$$

independent of whether the eigenvalues of the matrix $\hat{J}$ are distinct or not. According to (6), the equations'system (19) has the form:

$$
\sum_{\nu=0}^{q+1} C_{\nu, i} \lambda_{i}^{\nu}=0, \quad i=1,2, \ldots, m,
$$

where

$$
C_{\nu, i} \text { is generally a complex value. }
$$

Due to Wilf's definition [1]: The numerical integration method (4) is said to be stable if the roots of (20) for all $i=1,2, \ldots, m$ are inside the unit circle in the complex plane, so that the error introduced at any stage tends to decay than build up.

In this connection we state a theorem of Schur [5]:

The zero points of the polynomial:

$$
\mathrm{f}(\lambda) \equiv \mathrm{C}_{0}+\mathrm{C}_{1} \lambda+\ldots+\mathrm{C}_{\mathbf{q}+1} \lambda_{\mathbf{q}+1}
$$


are all inside the unit circle, if and only if the Hermitian form:

$$
\left.\mathrm{H}=\sum_{\mathrm{l}=0}^{\mathrm{q}}\left|\overline{\mathrm{C}}_{\mathrm{q}+1} \lambda_{1}+\overline{\mathrm{C}}_{\mathrm{q}} \lambda_{\mathrm{l}+1}+\ldots+\overline{\mathrm{C}}_{\mathrm{l}+1} \lambda_{\mathrm{q}}\right| 2\left|\mathrm{C}_{0} \lambda_{1}+\mathrm{C}_{1} \lambda_{\mathrm{l}+1}+\ldots+\mathrm{C}_{\mathrm{q}-1} \lambda_{\mathrm{q}}\right|^{2}\right\}
$$

is positive definite."

The quadratic form $H$ can be formed as follows:

$$
\begin{gathered}
H=\sum_{l=0}^{q}\left\{\sum_{r=0}^{q-l}\left(\left|C_{q+1-r}\right|^{2}-\left|C_{r}\right|^{2}\right)\left|\lambda_{l+r}\right|^{2}\right. \\
\left.+2 R_{e} \sum_{j=0}^{q-l} \sum_{k=j+1}^{q-l}\left(\bar{C}_{q+1-j} C_{q+1-k}-C_{j} \bar{C}_{k}\right) \lambda_{l+j} \bar{\lambda}_{l+k}\right\} \\
=\sum_{l=0}^{q}\left\{\sum_{s=l}^{q}\left(\left|C_{q+l+1-s}\right|^{2}-\left|C_{s-l}\right|^{2}\right)\left|\lambda_{s}\right|^{2}\right. \\
\left.+2 R_{e} \sum_{r=l}^{q} \sum_{s=r+1}^{q}\left(\bar{C}_{q+l+1-r} C_{q+l+1-s}-C_{r-l} \bar{C}_{s-l}\right) \lambda_{r} \bar{\lambda}_{s}\right\} \\
=\sum_{s=0}^{q}\left|\lambda_{s}\right|^{2} \sum_{l=0}^{s}\left(\left|C_{q+l+1-s}\right|^{2}-\left|C_{s-l}\right|^{2}\right) \\
+2 R_{e} \sum_{r=0}^{q} \sum_{s=r+1}^{q}\left\{\lambda_{r} \bar{\lambda}_{s} \sum_{l=0}^{s}\left(\bar{C}_{q+l+1-r} C_{q+l+1-s}-C_{r-l} \bar{C}_{s-l}\right)\right\}
\end{gathered}
$$

With

$$
A_{r s}=\sum_{l=0}^{\min (r, s)}\left\{\bar{C}_{q+l+1-r} C_{q+l+1-s}-C_{r-l} \bar{C}_{s-l}\right\}, \quad r, s=0,1, \ldots, q
$$

we have directly the form:

$$
H=\sum_{r, s=0}^{q} A_{r s} \lambda_{r} \bar{\lambda}_{s}
$$

For the system of differential equations (1) we have the Hermitian forms

$$
H^{(i)}=\sum_{r, s=0}^{g} A_{r s}^{(i)} \lambda_{r} \bar{\lambda}_{s}, \quad i=1,2, \ldots, m .
$$

Therefore $A_{r s}^{(i)}$ has the form (5) and accordingly the integration formula (4) is stable, if the Hermitian forms $H^{(i)}$ are positive definite for all $i$, so that theorem (1) is proved.

SPECIAL CASE: For $m=1$, we have one single differential equation in the form $y^{\prime}=f(x ; y)$. The Jacobian matrix reduces to one single element $\partial f / \partial y$ and $\kappa=-h \partial f / \partial y$ is a real value. The stability criterion reduces to Wilf Stability Criterion [1].

\section{Simple Examples}

EXAmPle 1:

$$
Y_{n+1}=Y_{n}+h Y_{n}^{\prime}, T_{2}=\frac{1}{2} h^{2} Y^{\prime \prime}\left(x_{n}\right)+O\left(h^{3}\right), \quad \text { (Euler-Cauchy method). }
$$

${ }^{2} \bar{C}$ means the conjugate complex value of 
With $q=0, a_{0}=1, a_{1}=-1$ and $b_{0}=1$ we have from (5) and (6):

$$
A_{00}^{(i)}=\left|C_{1, i}\right|^{2}-\left|C_{0, i}\right|^{2}=1-\left|\kappa_{i}-1\right|^{2} .
$$

Thus $A_{00}^{(i)}>0, i=1,2, \ldots, m$, if and only if $h \rho_{i}$ is inside the circle $\left|h \rho_{i}+1\right|=1$ (fig. 1) and so the integration method (21) is stable.

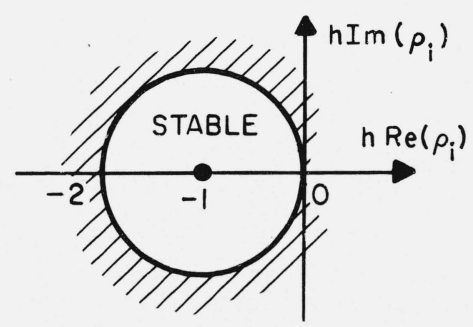

FigURE 1

EXAMPLE 2:

$$
\begin{gathered}
Y_{n+1}=-\frac{1}{3} Y_{n-1}+\frac{4}{3} Y_{n}+\frac{2}{3} h Y_{n}^{\prime}, \\
T_{2}=\frac{2}{3} h^{2} Y^{\prime \prime}\left(x_{n}\right)+O\left(h^{3}\right) .
\end{gathered}
$$

With $q=1, a_{-1}=-\frac{1}{3}, a_{0}=\frac{4}{3}, a_{1}=-1$ and $b_{0}=\frac{2}{3}$, we get from (5) and (6):

$$
\begin{gathered}
A_{00}^{(i)}=\left|C_{2, i}\right|^{2}-\left|C_{0, i}\right|^{2}=A_{11}^{(i)}, \\
A_{01}^{(i)}=\bar{C}_{2, i} C_{1, i}-C_{0, i} \bar{C}_{1, i}=\bar{A}_{10}^{(i)}, \\
C_{0, i}=\frac{1}{3}, C_{1, i}=\frac{2}{3}\left(\kappa_{i}-2\right), C_{2, i}=1 .
\end{gathered}
$$

The determinants:

$$
\begin{aligned}
& D_{1}^{(i)}=A_{00}^{(i)}=\frac{8}{9} \\
& D_{2}^{(i)}=\left(A_{00}^{(i)}\right)^{2}-\left|A_{01}^{(i)}\right|^{2}=\frac{4}{81}\left\{16-\left|3 \kappa_{i}-\bar{\kappa}_{i}-4\right|^{2}\right\}
\end{aligned}
$$

With $\kappa_{i}=-\left(\alpha_{i}+\sqrt{-1} \beta_{i}\right)$ it follows:

$$
D_{2}^{(i)}=-\frac{16}{81}\left\{\left(\alpha_{i}+2\right)^{2}+4 \beta_{i}^{2}-4\right\}>0
$$

if and only if $h \rho_{i}$ is inside the ellipse $\frac{\left(\alpha_{i}+2\right)^{2}}{4}+\beta_{i}^{2}=1$ (fig. 2) and so the integration method (22) is stable. 


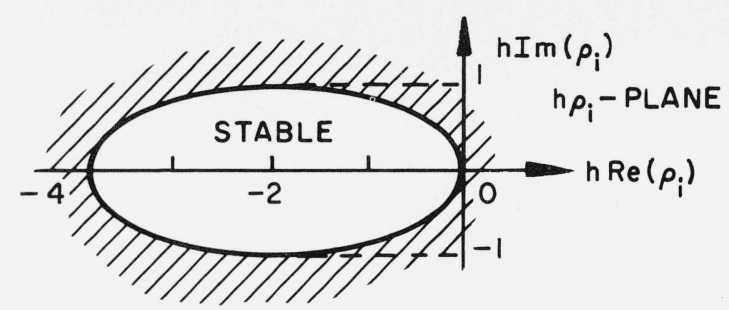

FIGURE 2

EXAmple 3:

$$
Y_{n+1}=Y_{n}+\frac{h}{2}\left(Y_{n}^{\prime}+Y_{n+1}^{\prime}\right), T_{3}=-\frac{1}{2} Y^{\prime \prime \prime}\left(x_{n}\right)+O\left(h^{4}\right)
$$

(Trapezoidal formula).

With $q=0, a_{0}=1, a_{1}=-1$ and $b_{0}=b_{1}=\frac{1}{2}$ we get from (5) and (6):

$$
A_{00}^{(i)}=\left|C_{1, i}\right|^{2}-\left|C_{0, i}\right|^{2}=\left|\frac{1}{2} \kappa_{i}-1\right|^{2}-\left|\frac{1}{2} \kappa_{i}+1\right|^{2}=2 \operatorname{Re}\left(\kappa_{i}\right) .
$$

We have $A_{00}^{(i)}>0$ if and only if $\operatorname{Re}\left(\rho_{i}\right)<0$, for $h>0$, and so the integration method (23) is stable.

EXAMPLE 4:

\section{More Difficult Examples}

$$
Y_{n+1}=\frac{1}{5} Y_{n-1}+\frac{4}{5} Y_{n}+\frac{2}{5} h\left(2 Y_{n}^{\prime}+Y_{n+1}^{\prime}\right), T_{4}=-\frac{1}{30} h^{4} Y^{\mathrm{v}}\left(x_{n}\right)+O\left(h^{5}\right)
$$

With $q=1, a_{-1}=\frac{1}{5}, a_{0}=\frac{4}{5}, a_{1}=-1, b_{0}=\frac{4}{5}$ and $b_{1}=\frac{2}{5}$ we get from (5) and (6):

$$
\begin{aligned}
A_{00}^{(i)} & =\left|C_{2, i}\right|^{2}-\left|C_{0, i}\right|^{2}=A_{11}^{(i)}, \\
A_{01}^{(i)} & =\overline{C_{2, i}} C_{1, i}-C_{0, i} \overline{C_{1}, i}=\overline{A_{10}^{(i)},} \\
C_{0, i} & =-\frac{1}{5}, C_{1, i}=\frac{4}{5}\left(\kappa_{i}-1\right), C_{2, i}=\frac{1}{5}\left(2 \kappa_{i}+5\right) .
\end{aligned}
$$

We have

$$
D_{1}^{(i)}=A_{00}^{(i)}=\frac{1}{25}\left\{\left|2 \kappa_{i}+5\right|^{2}-1\right\}>0
$$

if and only if $h \rho_{i}$ is outside the circle $\left|h \rho_{i}-2,5\right|=0,5$ (fig. 3), this means, the circle is the region of instability.

Further:

$$
\begin{aligned}
D_{2}^{(i)} & =\left\{A_{00}^{(i)}\right\}^{2}-\left|A_{01}^{(i)}\right|^{2} \\
& =\frac{1}{625}\left\{\left|2 \kappa_{i}+5\right|^{2}-1\right\}^{2}-\frac{16}{625}\left|\left(2 \bar{\kappa}_{i}+5\right)\left(\kappa_{i}-1\right)+\bar{\kappa}_{i}-1\right|^{2} \\
& =\frac{16}{625}\left\{\left\{\left|\kappa_{i}\right|^{2}+5 \operatorname{Re}\left(\kappa_{i}\right)+6\right\}^{2}-\left.|2| \kappa_{i}\right|^{2}+5 \kappa_{i}-\bar{\kappa}_{i}-\left.6\right|^{2}\right\} .
\end{aligned}
$$




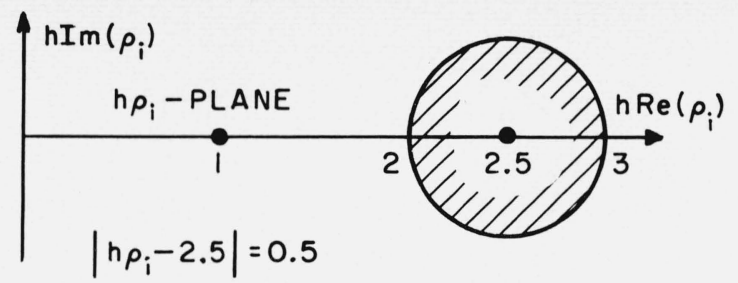

FigURE 3

With $\kappa_{i}=-\left(\alpha_{i}+\sqrt{-1} \beta_{i}\right)$ we get $D_{2}^{(i)}>0$ if

$$
\left\{\left(\alpha_{i}-2\right)\left(\alpha_{i}-3\right)+\beta_{l}^{2}\right\}^{2}>4\left\{\left\{\left(\alpha_{i}-3\right)\left(\alpha_{i}+1\right)+\beta_{l}^{2}\right\}^{2}+9 \beta_{l}^{2}\right\} \text {. }
$$

The inequalities $D_{v}^{(i)}>0, \nu=1,2$, are satisfied simultaneously, i.e., the integration formula (24) is stable if $h \rho_{i}$ is inside the region $R$ (fig. 4$)^{3}$

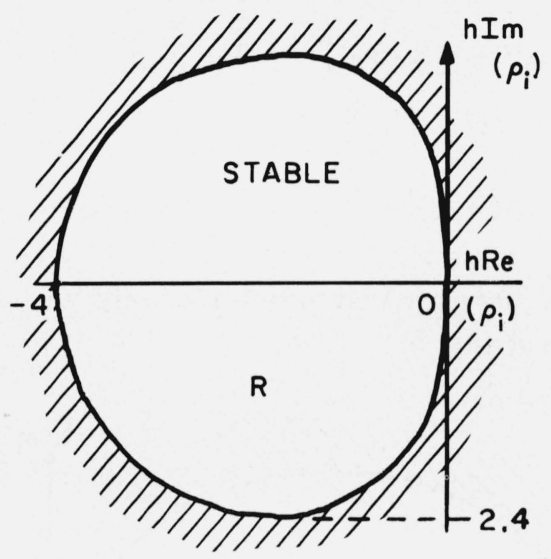

Figure 4

EXAMPle 5:

$$
Y_{n+1}=Y_{n}+\frac{h}{12}\left(-Y_{n-1}^{\prime}+8 Y_{n}^{\prime}+5 Y_{n+1}^{\prime}\right), \quad T_{4}=-\frac{h^{4}}{4 !} Y^{\mathrm{IV}}\left(x_{n}\right)+O\left(h^{5}\right) .^{4}
$$

With $q=1, a_{0}=1, a_{1}=-1, b_{-1}=-\frac{1}{12}, b_{0}=\frac{2}{3}$ and $b_{1}=\frac{5}{12}$ we get from (5) and (6):

$$
\begin{aligned}
A_{00}^{(i)} & =\left|C_{2, i}\right|^{2}-\left|C_{0, i}\right|^{2} \\
& =\frac{1}{6}\left\{\left|\kappa_{i}\right|^{2}+5 \operatorname{Re}\left(\kappa_{i}\right)+6\right\}=A_{11}^{(i)}, \\
A_{01}^{(i)} & =\bar{C}_{2, i} C_{1, i}-C_{0, i} \bar{C}_{1, i} \\
& =\frac{1}{12}\left(4\left|\kappa_{i}\right|^{2}+7 \kappa_{i}-5 \kappa_{i}-12\right)=\bar{A}_{10}^{(i)}, \\
C_{0, i} & =-\frac{1}{12} \kappa_{i},
\end{aligned}
$$

${ }^{3}$ The regions of stability (figs. 4, 5, and 6) are determined by means of the electronic digital computer $Z 22$.

${ }^{4}$ This formula is given by Southard and Yowell [6]. 


$$
\begin{aligned}
C_{1, i} & =\frac{1}{3}\left(2 \kappa_{i}-3\right), \\
C_{2, i} & =\frac{1}{12}\left(5 \kappa_{i}+12\right) .
\end{aligned}
$$

We have

$$
\begin{aligned}
D_{1}^{(i)} & =\frac{1}{6}\left\{\left|\kappa_{i}\right|^{2}+5 \operatorname{Re}\left(\kappa_{i}\right)+6\right\} \\
& =\frac{1}{6}\left\{\left|\kappa_{i}+2,5\right|^{2}-0,25\right\}>0
\end{aligned}
$$

if $h \rho_{i}$ is outside the circle $\left|h \rho_{i}-2,5\right|=0,5$ (fig. 3).

Further:

$$
\begin{aligned}
D_{i}^{(i)} & =\left\{A_{00}^{(i)}\right\}^{2}-\left|A_{0}^{(i)}\right|^{2} \\
& =\frac{1}{144}\left\{4\left\{\left|\kappa_{i}\right|^{2}+5 \operatorname{Re}\left(\kappa_{i}\right)+6\right\}^{2}-\left.|4| \kappa_{i}\right|^{2}+7 \kappa_{i}-5 \bar{\kappa}_{i}-\left.12\right|^{2}\right\} .
\end{aligned}
$$

With $\kappa_{i}=-\left(\alpha_{i}+\sqrt{-1} \beta_{i}\right)$ we get $\mathrm{D}_{2}^{(i)}>0$, if

$$
\left\{\left(\alpha_{i}-2\right)\left(\alpha_{i}-3\right)+\beta_{i}^{2}\right\}^{2}>\left\{\left(\alpha_{i}-2\right)\left(2 \alpha_{i}+3\right)+2 \beta_{i}^{2}\right\}^{2}+36 \beta_{i}^{2} .
$$

The inequalities $D_{\nu}^{(i)}>0, \nu=1,2$ are satisfied simultaneously, i.e., the integration formula (25) is stable, if $h \rho_{i}$ is inside the region $R$ (fig. 5)

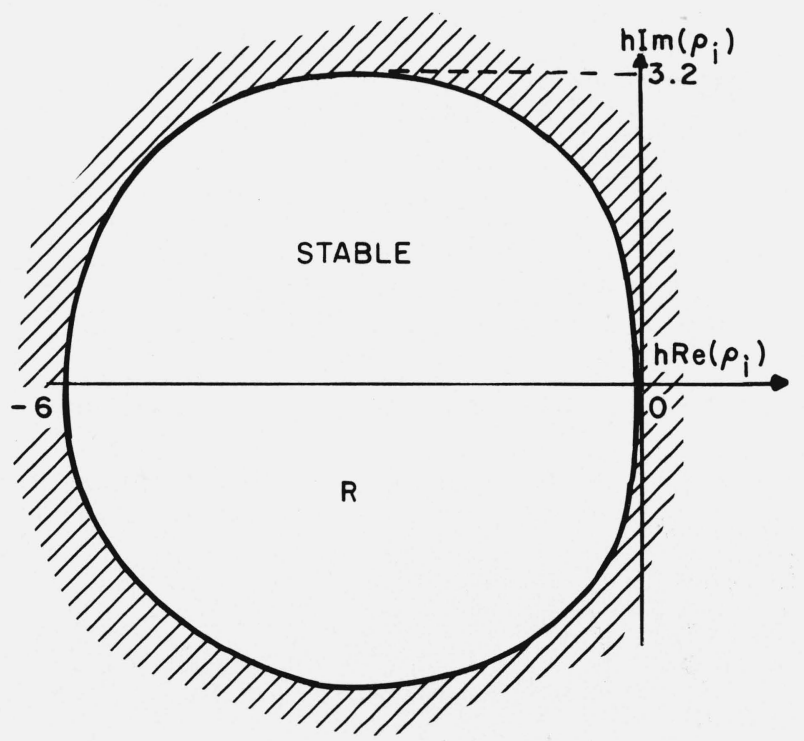

FigURE 5

EXAMPLE 6:

$$
Y_{n+1}=-\frac{1}{9} Y_{n-2}+\frac{1}{9} Y_{n-1}+Y_{n}+\frac{2}{27}\left(-4 Y_{n-1}^{\prime}+11 Y_{n}^{\prime}+5 Y_{n+1}^{\prime}\right), T_{5}=\frac{-19}{810} h^{5} Y^{V}\left(x_{n}\right)+0\left(h^{6}\right)
$$

(Hamming [2]). 
With $q=2, a_{-2}=-\frac{1}{9}, a_{-1}=\frac{1}{9}, a_{0}=1, a_{1}=-1$,

$$
b_{-1}=-\frac{8}{27}, b_{0}=\frac{22}{27}, b_{1}=\frac{10}{27} \text { we get from (5): }
$$

$C_{0, i}=\frac{1}{9}, C_{1, i}=-\frac{1}{27}\left(8 \kappa_{i}+3\right)$,

$C_{2, i}=\frac{1}{27}\left(22 \kappa_{i}-27\right), C_{3, i}=\frac{1}{27}\left(10 \kappa_{i}+27\right)$.

The elements of the Hermitian Matrix follow

$$
\begin{aligned}
& A_{00}^{(i)}=\bar{C}_{3, i} C_{3, i}-C_{0, i} \bar{C}_{0, i}=A_{22}^{(i)}, \\
& A_{01}^{(i)}=\bar{C}_{3, i} C_{2, i}-C_{0, i} \bar{C}_{1, i}=\bar{A}_{10}^{(i)}=A_{12}^{(i)}=\bar{A}_{21}^{(i)}, \\
& A_{02}^{(i)}=\bar{C}_{3, i} C_{1, i}-C_{0, i} \bar{C}_{2, i}=\bar{A}_{20}^{(i)}, \\
& A_{11}^{(i)}=\bar{C}_{3, i} C_{3, i}+\bar{C}_{2, i} C_{2, i}-C_{1, i} \bar{C}_{1, i}-C_{0, i} \bar{C}_{0, i} .
\end{aligned}
$$

We have

$$
\begin{aligned}
& D_{1}^{(i)}=A_{00}^{(i)}=\left|C_{3, i}\right|^{2}-\left|C_{0, i}\right|^{2} \\
& =\frac{1}{(27)^{2}}\left\{\left|10 \kappa_{i}+27\right|^{2}-9\right\}>0 \text {, if } h \rho_{i} \text { is outside the circle }\left|h \rho_{i}-2,7\right|=0,3 \text {, (fig. 6). }
\end{aligned}
$$

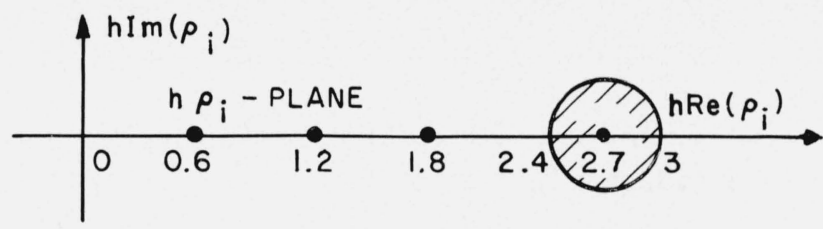

$$
\begin{aligned}
& \left|h p_{i}-2.7\right|=0.3
\end{aligned}
$$

Figure 6

\section{Further}

$$
\begin{aligned}
D_{2}^{(i)}=A_{00}^{(i)} A_{11}^{(i)}-\left|A_{01}^{(i)}\right|^{2}= & \left\{\left|C_{3, i}\right|^{2}-\left|C_{0, i}\right|^{2}\right\}\left\{\left|C_{3, i}\right|^{2}+\left|C_{2, i}\right|^{2}\right. \\
\left.-\left|C_{1, i}\right|^{2}-\left|C_{0, i}\right|^{2}\right\}-\left|\bar{C}_{3, i} C_{2, i}-C_{0, i} \bar{C}_{1, i}\right|^{2}=\left\{\left|C_{3, i}\right|^{2}-\left|C_{0, i}\right|^{2}\right\}^{2}-\left|\bar{C}_{3, i} C_{1, i}-C_{0, i} \bar{C}_{2, i}\right|^{2} & \\
= & \frac{16}{(27)^{4}}\left[25\left\{5\left(\alpha_{i}^{2}+\beta_{i}^{2}\right)-27 \alpha_{i}+36\right\}^{2}-4\left\{\left\{10\left(\alpha_{i}^{2}+\beta_{i}^{2}\right)-39 \alpha_{i}\right\}^{2}+225 \beta_{i}^{2}\right\}\right] .
\end{aligned}
$$

From this it can be proved, that $\mathrm{D}_{2}^{(i)}>0$ for $\alpha_{i}<0$. Finally we get:

$D_{3}^{(i)}=\left|\begin{array}{lll}\bar{A}_{00}^{(i)} & A_{01}^{(i)} & A_{02}^{(i)} \\ \bar{A}_{01}^{(i)} & A_{11}^{(i)} & A_{01}^{(i)} \\ \bar{A}_{02}^{(i)} & \bar{A}_{01}^{(i)} & A_{00}^{(i)}\end{array}\right|$

$$
=A_{11}^{(i)}\left\{\left(A_{00}^{(i)}\right)^{2}-\left|A_{02}^{(i)}\right|^{2}\right\}-2 A_{00}^{(i)}\left|A_{01}^{(i)}\right|^{2}+2 \operatorname{Re}\left(\bar{A}_{02}^{(i)}\left(A_{01}^{(i)}\right)^{2}\right) .
$$


Put

$$
\begin{array}{r}
A_{11}^{(i)}\left\{\left(A_{00}^{(i)}\right)^{2}-\left|A_{02}^{(i)}\right|^{2}\right\}=\frac{32}{(27)^{6}} f_{1}\left(\alpha_{i}, \beta_{i}\right), \\
-2 A_{00}^{(i)}\left|A_{01}^{(i)}\right|^{2}=\frac{32}{(27)^{6}} f_{2}\left(\alpha_{i}, \beta_{i}\right), \\
2 \operatorname{Re}\left(\bar{A}_{02}^{(i)}\left(A_{01}^{(i)}\right)^{2}\right)=\frac{32}{(27)^{6}} f_{3}\left(\alpha_{i}, \beta_{i}\right),
\end{array}
$$

where

$$
\begin{gathered}
f_{1}\left(\alpha_{i}, \beta_{i}\right)=4\left[65\left(\alpha_{i}^{2}+\beta_{i}^{2}\right)+87 \alpha_{i}+180\right]\left[2 5 \left\{5\left(\alpha_{i}^{2}+\beta_{i}^{2}\right)\right.\right. \\
\left.\left.-27 \alpha_{i}+36\right\}^{2}-4\left\{\left(10\left(\alpha_{i}^{2}+\beta_{i}^{2}\right)-39 \alpha_{i}\right)^{2}+225 \beta_{i}^{2}\right\}\right], \\
f_{2}\left(\alpha_{i}, \beta_{i}\right)=-20\left[5\left(\alpha_{i}^{2}+\beta_{i}^{2}\right)-27 \alpha_{i}+36\right]\left[\left\{55\left(\alpha_{i}^{2}+\beta_{i}^{2}\right)\right.\right. \\
\left.\left.-87 \alpha_{i}-180\right\}^{2}+44100 \beta_{i}^{2}\right], \\
f_{3}\left(\alpha_{i}, \beta_{i}\right)=-8\left[( 1 0 ( \alpha _ { i } ^ { 2 } + \beta _ { i } ^ { 2 } ) - 3 9 \alpha _ { i } ) \left\{\left(55\left(\alpha_{i}^{2}+\beta_{i}^{2}\right)-87 \alpha_{i}\right.\right.\right. \\
\left.\left.-180)^{2}-44100 \beta_{i}^{2}\right\}+6300 \beta_{i}^{2}\left(55\left(\alpha_{i}^{2}+\beta_{i}^{2}\right)-87 \alpha_{i}-180\right)\right] .
\end{gathered}
$$

From these we get $D_{3}^{(i)}>0$, if

$$
f_{1}\left(\alpha_{i}, \beta_{i}\right)+f_{2}\left(\alpha_{i}, \beta_{i}\right)+f_{3}\left(\alpha_{i}, \beta_{i}\right)>0 \text {. }
$$

The inequalities $D_{\nu}^{(i)}>0, \nu=1,2,3$ are satisfied simultaneously, i.e., the integration formula is stable if $h \rho_{i}$ is inside the region $R$ (fig. 7).

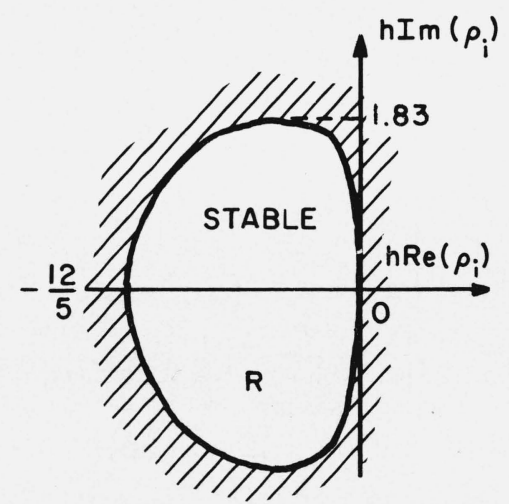

Figure 7

\section{References}

[1] Wilf, H. S., A Stability criterion for numerical integration, J. Assoc. Comp. Mach. V. 6, No. 3, 363-365 (July 1959).

[2] Hamming, R. W., Stable prediotor-corrector methods for ordinary differential equations, J. Assoc. Comp. Mach. V. 6, No. 1, P. 37-47 (Jan. 1959).

[3] Zurmühl, R., Matrizen, 3, Aufl., Berlin, (1961). 
[4] Nörlund, N. E., Vorlesungen über Differenzenrechnung, New York (1954).

[5] Schur, J. Über Potenzreihen, die in Innern des Einheitskreises beschränkt sind. J. Reine Angew. 147, 205-232 (1917). und 148, 122-145 (1918).

[6] Southerd and Yowell, Math. Tables Comp. 6, 253-4 (1952).

(Paper 71B2\&3-204) 